link to each other ${ }^{5}$ (similar to the situation depicted in Fig. 1a), whereas hubs in most biological networks avoid each other ${ }^{6}$ (see Fig. 1c). The correlation profile of the Internet is more complicated ${ }^{7}$ and reflects its hierarchical organization in which lowdegree 'user' nodes connect to mid-level Internet Service Providers with intermediate degrees, which in turn are connected to highly-connected global hubs.

Guimerà et al. ${ }^{3}$ now extend our understanding of these connectivity patterns. They advocate that the classification of nodes should not be based exclusively on their degree but also on their position with respect to the modules present in the network. Both hubs and less-connected nodes can be classified either as being peripheral (or provincial) - that is, its neighbours tend to belong to the same module - or as connectors, nodes that efficiently link different modules. This classification scheme captures the intuitive notion that it is not just the quantity but also the quality of neighbours that matters.

For biological networks this approach is related to an earlier classification scheme $e^{8}$ dividing highly connected proteins in physical interaction networks into 'party' and 'date' hubs. Party hubs interact with most of their partners at once (that is, they tend to belong to the same functional module), but date hubs prefer to meet their partners one at a time at different external or internal conditions. It is likely that party and date hubs in protein interaction networks ${ }^{8}$ roughly correspond to provincial and connector hubs in the more general definition of Guimerà and colleagues ${ }^{3}$.

The generalization of the classification scheme leads to important conclusions. First, in a whole series of real-world networks that Guimerà et al. ${ }^{1}$ looked at, intermodular connectivity patterns fully account for the observed degree-degree correlations (with the notable exception of the network formed by physical protein-protein interactions). Second, Guimerà and colleagues ${ }^{3}$ find statistically significant patterns in how different categories of nodes connect to each other (they call these patterns 'role-to-role connectivity profiles'). This extends and refines an earlier concept of the correlation profile $^{6,7}$ in that, for example, the 'repulsion' between hubs in protein interaction networks is shown to be limited to connector hubs and does not involve provincial hubs. Role-to-role connectivity profiles allow complex networks to be divided into two main classes. Intriguingly, the first class contains networks used for transport or flow of materials where conservation laws

apply - air-traffic and metabolic networks, for example - and the second class seems to be optimized for signalling and information transfer, such as in protein-protein interactions and the Internet.

Another important lesson learned from the work by Guimerà et al. ${ }^{3}$ is that to discover characteristic topological patterns in a complex network one needs to compare each case to a series of null-models of progressively increasing complexity. Whenever a higher-level topological property is studied, it makes sense to compare the real network with its randomized version ${ }^{7}$ preserving all known lower-level features such as the degree distribution or the extent of modularity. Only such a comparison ensures that overrepresentation of a higherlevel pattern does indeed constitute a new result, and is not just a direct consequence of some previously reported property.

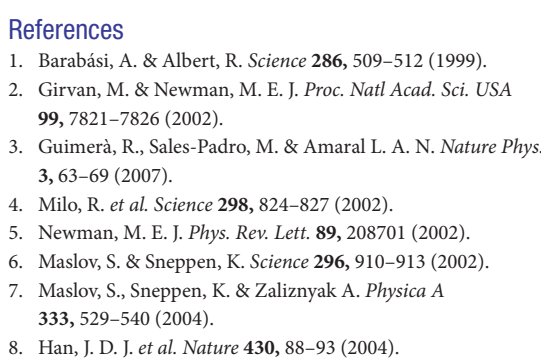

\title{
BIOPHYSICS
}

\section{Pushed to the limit}

Viruses come in neat packages. The genetic material of a virus, which is passed into a host cell during infection, is bound up inside a shell of proteins, known as a capsid.

William S. Klug and colleagues have made both a theoretical and a practical study of the behaviour of a virus capsid under pressure (Phys. Rev. Lett. 97, 228101; 2006). Their work underlines the importance of the Föppl-van Kármán (FvK) number - a dimensionless ratio of stretching to bending stiffness - in determining a capsid's resilience.

The FvK number is defined as $Y R^{2} / \kappa$, where $Y$ is the two-dimensional Young's modulus of the in-plane elasticity of the shell, $R$ is the radius of the shell and $\kappa$ is the out-of-plane bending modulus. The FvK number is already thought to be a factor in the morphology of capsids: if its value is lower than the so-called buckling threshold, the shell is almost spherical; if not, the shell is polyhedral.

According to Klug and colleagues' simulations, low FvK number $(\sim 100)$ also results in a linear force-indentation response, which breaks down only at very deep indentations. But for higher FvK values ( $>400)$, the relationship becomes nonlinear at lower indentations and discontinuities appear.

That behaviour is borne out in their experimental tests on an empty capsid of cowpea chlorotic mottle virus (CCMV, pictured), prodded using the tip of an atomic force microscope. The CCMV capsid has an outer diameter of 24-28 nm, and a shell thickness of 2-4 nm. At pH 6, its force-indentation response is linear up to an indentation equivalent to $70 \%$ of the capsid diameter - suggesting a low FvK number. At that point, the capsid becomes incompressible (the two inner surfaces are in contact), but the process is reversible, and the authors report minimal hysteresis over repeated measurements.

Previous work at $\mathrm{pH} 5$ told a different story: that nonlinearity kicks in at around $30 \%$ indentation, and a much higher FvK value is favoured. But the CCMV capsid is known to swell as the $\mathrm{pH}$ value of its surroundings is increased from 5 to 7 , and that could be behind the seeming 'softening' of the capsid. Energetic considerations using

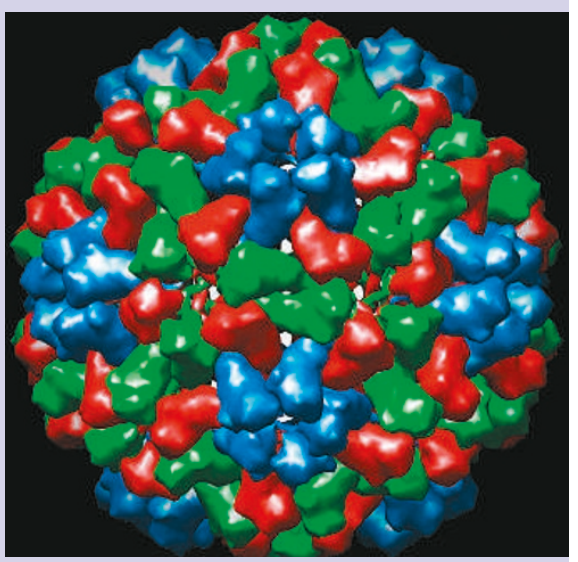

Ginzburg-Landau theory suggest a reduced effective Young's modulus comes into play, which in turn means a lower FvK value.

The change from high to low FvK value with the swelling doesn't, however, translate into a shape change, despite the earlier matching of FvK number to morphology. That, say Klug et al., means the FvK number is more a general indicator of elastic response than had been thought.

Alison Wright 